\title{
Oral bioavailability and hypoglycaemic activity of tolbutamide/cyclodextrin inclusion complexes
}

\author{
F. Veiga ${ }^{\mathrm{a}, *}$, C. Fernandes ${ }^{\mathrm{a}}$, F. Teixeira ${ }^{\mathrm{b}}$ \\ a Laboratório de Tecnologia Farmacêutica, Faculdade de Farmácia, Universidade de Coimbra, Rua do Norte, \\ 3000 Coimbra, Portugal \\ b Laboratório de Farmacologia, Faculdade de Medicina, Universidade de Coimbra, Rua do Norte, 3000 Coimbra, Portugal
}

Received 1 April 2000; accepted 11 May 2000

\begin{abstract}
The purpose of the present study was to evaluate the enhancement of tolbutamide (TBM) oral bioavailability and hypoglycaemic activity through complexation with $\beta$-cyclodextrin $(\beta-\mathrm{CD})$ and hydroxypropyl- $\beta$-cyclodextrin (HP- $\beta$ $\mathrm{CD}$ ). TBM and its freeze-dried inclusion complexes were administered to rabbits (New zealand breed; $n=6$ ), in a dose of $20 \mathrm{mg} / \mathrm{kg}$. TMB plasma levels were measured by HPLC and glucose levels were analysed according to Trinder (Trinder, P., 1969. Determination of glucose in blood using glucose oxidase with an alternative oxygen acceptor. Ann. Clin. Biochem. 6, 24-28). The pure drug attained a maximum of plasma concentration $\left(C_{\max }\right)$ of $18.58 \pm 3.27 \mu \mathrm{g} / \mathrm{ml}$ at $8.5 \mathrm{~h}\left(T_{\max }\right)$, whereas with inclusion complexes, $C_{\max }$ increased about two times and appeared at ca. $4 \mathrm{~h}$. $\mathrm{AUC}_{0-24}$ of complexes was about 1.6 times as much as that of the pure drug. Thus, the extent of oral absorption of TBM from inclusion complexes was significantly greater and faster when compared with drug alone. In addition, without cyclodextrins the maximum hypoglycaemic effect $\left(\mathrm{CVG}_{\max }\right)$ of TBM $(34.1 \%)$ was observed at $5.6 \mathrm{~h}\left(T g_{\max }\right) \cdot \mathrm{CVG}_{\max }$ of $\mathrm{TBM} / \beta-\mathrm{CD}$ and TBM/HP- $\beta-\mathrm{CD}$ inclusion complexes were $34.1 \%$ (at $6.5 \mathrm{~h}$ ) and $37.7 \%$ (at $5.1 \mathrm{~h}$ ), respectively. $\mathrm{AAC}_{0-24}$ of inclusion complexes was 1.4 times larger than that of pure drug. Hence, the oral administration of complexed TBM not only improved the drug absorption, but also the TBM hypoglycaemic activity. (C) 2000 Elsevier Science B.V. All rights reserved.
\end{abstract}

Keywords: Cyclodextrins; Tolbutamide; Inclusion complexes; Bioavailability; Hypoglycaemic activity

\section{Introduction}

Cyclodextrins (CDs) are cyclic oligosaccharides, containing six $(\alpha-C D)$, seven $(\beta-C D)$ or eight $(\gamma-$

\footnotetext{
* Corresponding author. Tel.: + 351-39-837850; fax: + 35139-837731.

E-mail address: fveiga@pop.ci.uc.pt (F. Veiga).
}

CD) $\alpha$-1,4-linked D-glucose units, with a hydrophilic outer surface and a hydrophobic cavity, in which may be included a great variety of 'guest' molecules of suitable size and shape, resulting in a stable association without formation of covalent bonds (Szejtli, 1990). In the pharmaceutical field this phenomenon has been extensively applied to enhance the solubility, dissolution rate and bioavailability of slightly soluble drugs in gas- 
trointestinal fluids (Vila-Jato et al., 1988; Soliman et al., 1997; Ventura et al., 1997; Dhanaraju et al., 1998; Özdemir and Ordu, 1998; Mura et al., 1999).

Among the cyclodextrins, $\beta-\mathrm{CD}$ is the most useful compound for drug complexation. However, the relatively low aqueous solubility of $\beta$-CD (about $1.8 \% \mathrm{w} / \mathrm{v}$, at $25^{\circ} \mathrm{C}$ ) suggested the use of chemically-modified CDs with different physical properties and inclusion behaviour. In particular, hydroxypropyl- $\beta$-cyclodextrin $\quad(\mathrm{HP}-\beta-\mathrm{CD})$ is widely used, because of its amorphous nature, high water solubility and solubilising power and low toxicity (Pitha and Pitha, 1985; Brewster, 1991).

Tolbutamide (TBM) is used as an oral hypoglycaemic agent. The drug is practically insoluble in water and its dissolution is supposed to be the rate-limiting step for its absorption from the gastrointestinal tract (Miralles et al., 1982). Several studies on commercial brands of tolbutamide dosage forms have shown marked variations in dissolution rate and bioavailability (Carminetsky, 1963; Varley, 1968; Ayres et al., 1984).

We previously reported (Veiga et al., 1996) that the solubility and dissolution rate of TBM is markedly increased due to complexation with $\beta$ $\mathrm{CD}$ and HP- $\beta-C D$. These preliminary studies suggested that the TBM oral bioavailability could be improved through complexation with these CDs.

The objective of the present study was to evaluate the influence of the complexation on the bioavailability and hypoglycaemic activity of tolbutamide after oral administration to rabbits of TBM/ $\beta-C D$ and TBM/HP- $\beta-C D$ complexes when compared with pure drug.

\section{Material and methods}

\subsection{Materials}

Tolbutamide (Sigma), $\beta$-CD (Roquette Frères) and HP- $\beta-C D$ with a molar substitution of 0.39 (Janssen) were used as received. All other reagents and solvents were of analytical grade.

\subsection{Inclusion complex preparation}

The inclusion complexes of $\mathrm{TBM} / \beta-\mathrm{CD}(1: 2)$ and TBM/HP- $\beta$-CD (1:1) were prepared by the freeze-drying method, previously described by Veiga et al. (1996). Stoichiometric quantities of TBM and CDs were dissolved in distilled water with a small amount of ammonia $25 \%$ to aid the active ingredient to dissolve. The solution was frozen by immersion in a shell freezer and freezedried over $24 \mathrm{~h}$ in a Lyph-lock 6 apparatus (Labconco). No trace of ammonium was detected in the resulting mixture with Nessler reagent.

\subsection{In vivo studies}

Male rabbits (New zealand breed) were used with an average weight of $3.5 \mathrm{~kg}$. They were kept on a standard diet (702 GR, Rico Gado) and made to fast for $12 \mathrm{~h}$ prior to experiments. At least 15 days were allowed to elapse between the administrations.

The three formulations (commercial tolbutamide, TBM $/ \beta-C D$ and TBM/HP- $\beta-C D$ inclusion complexes) with a dose of $20 \mathrm{mg} / \mathrm{kg}$ of tolbutamide or equivalent were previously suspended in $30 \mathrm{ml}$ of water. The aqueous suspensions were given by intragastric tubing. The administration scheme is given in Table 1 .

Table 1

Administration scheme of TBM alone, TBM/ $\beta-C D$ and TBM/HP- $\beta-C D$ inclusion complexes

\begin{tabular}{lllll}
\hline Animals & 1st Set & 2nd Set & 3rd Set & 4th Set \\
\hline Group I & Control & TBM & TBM/ $\beta-C D$ & TBM/HP- $\beta-C D$ \\
Group II & TBM & TBM $/ \beta-C D$ & TBM/HP- $\beta$-CD & Control \\
Group III & TBM $/ \beta-C D$ & TBM $/$ HP- $\beta-C D$ & Control & TBM \\
\hline
\end{tabular}


The blood samples $(1 \mathrm{ml})$ were collected over a period of $24 \mathrm{~h}$ from the marginal ear vein. EDTA was added to the blood samples to prevent clotting and subsequently, plasma was obtained by centrifugation at $2800 \mathrm{rev}$. $/ \mathrm{min}$ for $15 \mathrm{~min}$. The plasma samples were stored at $-30^{\circ} \mathrm{C}$ until analysis.

\subsection{Determination of TBM plasma levels}

The plasma levels of TBM were measured by high-performance liquid chromatography (HPLC).

\subsubsection{HPLC conditions}

HPLC equipment consisted of a Gilson 305 pump, Rheodyne injector (with a 20- $\mu$ l loop), Gilson 117 UV spectrometric detector, Kipp and Zonen register and a Li-Chrospher RP 18 reversed-phase column $(10 \mu \mathrm{m}$ particle size, $250 \times$ $4.6 \mathrm{~mm}$ i.d., Jones). A guard column (Jones), similarly packed, was used before the analytical column. The mobile phase was prepared by degassing and filtering (through $0.45-\mu \mathrm{m}$ membranes, Millipore) a mixture of acetonitrile and $0.05 \%$ phosphoric acid (45:55). The chromatograph was operated at a flow rate of 1.5 $\mathrm{ml} / \mathrm{min}$ and the eluent was monitored spectrophotometrically at the UV maximum of TBM (226 $\mathrm{nm})$. The sample injection size was $20 \mu \mathrm{l}$. All the determinations were performed at room temperature $\left(20 \pm 2^{\circ} \mathrm{C}\right)$.

\subsubsection{HPLC method validation}

Standard curves for TBM were developed in the mobile phase and in the rabbit plasma, for the TBM range of $1-60 \mu \mathrm{g} / \mathrm{ml}$. For construction of standard curves in plasma, different volumes (5$300 \mu \mathrm{l})$ of a $100 \mu \mathrm{g} / \mathrm{ml}$ TBM methanolic solution were used. After methanol evaporation, $200 \mu \mathrm{l}$ of rabbit plasma and $500 \mu \mathrm{l}$ of acetonitrile were added. The mixtures were vortexed for $10 \mathrm{~s}$ and subsequently centrifuged at $2500 \mathrm{rev}$./min for 1 $\min$.

TBM recovery from plasma was determined with two concentrations $(n=3)$ by comparing the peak areas obtained for TBM plasma samples and those of TBM phase mobile solutions. Recoveries of TBM from plasma samples with 20 and 40 $\mu \mathrm{g} / \mathrm{ml}$ concentrations were 98.7 and $99.1 \%$, respectively.

Validation of HPLC method was evaluated by linearity, precision (repeatability and intermediate precision), accuracy and limit of detection. The calibration curve of TBM was linear over the concentration range of $1-60 \mu \mathrm{g} / \mathrm{ml}(r>0.999)$. The repeatability was evaluated by six replicate determinations of solutions with two different concentrations of TBM. The RSD obtained for 20 and $40 \mu \mathrm{g} / \mathrm{ml} \mathrm{TBM}$ solutions were 1.9 and $1.63 \%$, respectively. The intermediate precision was determined by analysing these solutions in five consecutive days. The RSD were $2.86 \%(20 \mu \mathrm{g} / \mathrm{ml})$ and $2.27 \%(40 \mu \mathrm{g} / \mathrm{ml})$. The accuracy, expressed as recovery percentage of TBM from plasma, was assessed by spiking plasma samples with TBM in $20 \mu \mathrm{g} / \mathrm{ml}$ concentration. The mean recovery for six replicate plasma samples was compared with the absolute recovery $(100 \%)$ and the statistical analysis, performed with Student's $t$-test, demonstrated that this difference was statistically not significant $(P<0.05)$. The detection limit for the assay was estimated at $1 \mu \mathrm{g} / \mathrm{ml}$.

\subsection{Determination of plasma glucose}

The glucose levels were analysed according to Trinder (1969) using the glucose enzymatic reagent system (glucose oxidase/peroxidase method), in an auto-analyser (Pronto BPC Biosed).

Plasma glucose levels (Glyc) were transformed into the plasma glucose variation coefficient (GVC) according to the expression:

GVC $=\frac{\text { Glyc. at } t-\text { Glyc. at } t=0}{\text { Glyc. at } t=0} \times 100$

\subsection{Pharmacokinetics and pharmacodynamics}

The pharmacokinetic and pharmacodynamic parameters were calculated using a statistical and analytical computer programme (PKCALC, Software Arts).

The areas under TBM plasma concentrationtime curves $\left(\mathrm{AUC}_{0-24}\right.$ and $\left.\mathrm{AUC}{ }_{0-\infty}\right)$, calculated 


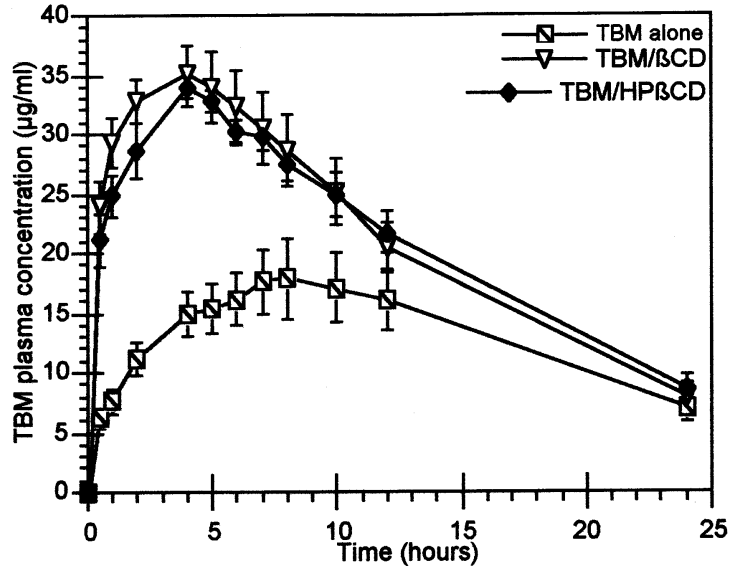

Fig. 1. TBM plasma concentration-time profiles after administration of TBM, TBM $/ \beta-\mathrm{CD}$ and TBM/HP- $\beta-\mathrm{CD}$ inclusion complexes (equivalent to TBM $20 \mathrm{mg} / \mathrm{kg}$ ) to rabbits $(n=6)$. Each point represents the mean \pm S.D.

by the linear trapezoidal method, the maximum plasma concentration of the $\operatorname{drug}\left(C_{\max }\right)$, the time to reach $C_{\max }\left(T_{\max }\right)$ and TBM elimination halflife $\left(t_{1 / 2}\right)$ were chosen as parameters for pharmacokinetic evaluation. The relative bioavailability between the inclusion complexes and commercial drug was calculated from the ratio of their AUC values.

The area above the curve of glucose variation coefficient $(\mathrm{GVC})$ versus time $\left(\mathrm{AAC}_{0-24}\right)$, calculated by linear trapezoidal rule, the percent maximum of GVC depression $\left(\mathrm{GVC}_{\max }\right)$ and the time to reach $\mathrm{GVC}_{\max }\left(T g_{\max }\right)$ were selected as parameters for pharmacodynamic evaluation.

Statistical analysis of results was performed using one-way analysis of variance (ANOVA) and the least-significant difference test (LSD) at 95\% of confidence $(P<0.05)$.

\section{Results and discussion}

Plasma concentration-time profiles for TBM after oral administration of drug alone to rabbits, $\mathrm{TBM} / \beta-\mathrm{CD}$ and TBM/HP- $\beta-\mathrm{CD}$ inclusion complexes (equivalent dose of TBM $20 \mathrm{mg} / \mathrm{kg}$ ) are illustrated in Fig. 1. The pharmacokinetic parameters derived from the plasma data are presented in Table 2. The plasma levels of TBM after administration of inclusion complexes were clearly faster and higher than those achieved with an equal TBM dose given alone. In particular, the $C_{\text {max }}$ after administration of TBM alone was observed at $8.5 \mathrm{~h}$; on the other hand, TBM complexes resulted in the rapid appearance of TBM in plasma, attaining the $C_{\max }$ after ca. $4 \mathrm{~h}$. In addition, the value of $C_{\max }$ for the complexes (36.26 \pm 2.17 and $34.99 \pm 1.36 \mu \mathrm{g} / \mathrm{ml}$ to $\beta-C D$ and HP- $\beta$-CD complexes, respectively) was about two times greater that of TMB alone $(18.58 \pm 3.27$ $\mu \mathrm{g} / \mathrm{ml})$. The differences in the mean of $C_{\max }$ and $T_{\max }$ between the TBM and the complexes were statistically significant $(P<0.05)$, but no significant differences in these plasma parameters were observed between the two types of complexes. A similar trend was observed with the $t_{1 / 2}$, in which there was a significant difference $(P<0.05)$ only between TBM alone and the inclusion complexes. The high $t_{1 / 2}$ values can be attributed to the strong and extensive binding of TBM to plasma proteins, in animals and humans (about 96\%) (Tillement, 1983).

The $\mathrm{AUC}_{0-24}$ of complexes was found to be about 1.6-fold greater than the TBM alone. Similarly, $\mathrm{AUC}_{0-\infty}$ for the complexes was 1.4 times as much as that from reference TBM. The statistical

Table 2

Pharmacokinetic parameters and relative bioavailability of TBM after oral administration of TBM, TBM/ $\beta-\mathrm{CD}$ and TBM/HP- $\beta-\mathrm{CD}$ inclusion complexes (equivalent to $\mathrm{TBM} 20 \mathrm{mg} / \mathrm{kg}$ ) to rabbits ${ }^{\mathrm{a}}$

\begin{tabular}{lllllrl}
\hline System & $C_{\max }(\mu \mathrm{g} / \mathrm{ml})$ & $T_{\max }(\mathrm{h})$ & \multicolumn{1}{l}{$\begin{array}{l}\mathrm{AUC}_{0-24} \\
(\mu \mathrm{g} \mathrm{h} / \mathrm{ml})\end{array}$} & $\begin{array}{l}\mathrm{AUC}_{0-\infty} \\
(\mu \mathrm{g} \mathrm{h} / \mathrm{ml})\end{array}$ & $T_{1 / 2}(\mathrm{~h})$ & $\begin{array}{l}\text { Rel. bioavailability } \\
(\%)\end{array}$ \\
\hline TBM & $18.58 \pm 3.27$ & $8.50 \pm 1.15$ & $312.95 \pm 47.21$ & $443.96 \pm 63.33$ & $12.48 \pm 1.33$ & \\
$\begin{array}{l}\beta-C D \text { complex } \\
\text { HP- } \beta-C D\end{array}$ & $36.26 \pm 2.17$ & $3.83 \pm 0.65$ & $516.26 \pm 45.05$ & $621.62 \pm 52.73$ & $8.79 \pm 0.47$ & 165.00 \\
$\quad$ complex & $34.99 \pm 1.36$ & $4.00 \pm 0.45$ & $510.76 \pm 28.22$ & $654.63 \pm 62.42$ & $10.22 \pm 1.19$ & 163.21 \\
\hline
\end{tabular}

\footnotetext{
${ }^{\text {a }}$ Each value represents the mean \pm S.D. for six rabbits.
} 


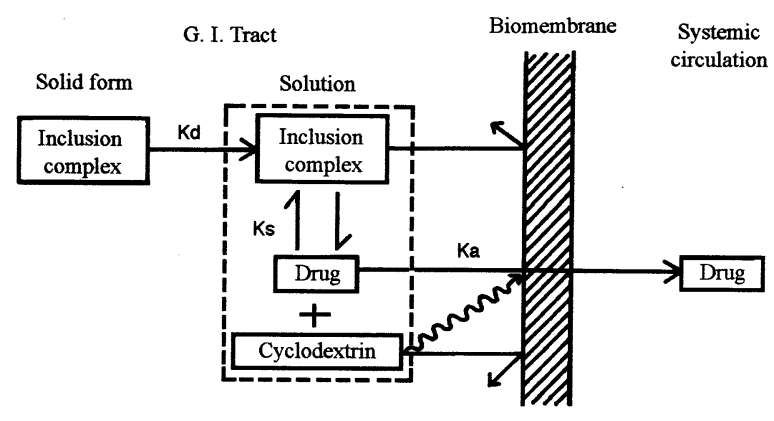

Fig. 2. Drug absorption mechanism involving cyclodextrin complexation, adapted from Uekama and Otagiri (1987).

study demonstrated that there were significant differences in these areas between TBM and the complexes $(P<0.05)$, while the differences of the two complexes were statistically insignificant. This enhancement in TBM AUCs can be attributed to the increase in TBM solubility and dissolution rate upon complexation with CDs. However, this improvement in TBM bioavailability was clearly independent of the cyclodextrin employed in complexation, although the solubility of TMB was 2.5 -fold higher in HP- $\beta$-CD complexes than in the $\beta$-CD complexes, as previously reported (Veiga et al., 1996).

Since only the free form of the drug can pass through the lipid barrier of the gastrointestinal tract, after oral administration of cyclodextrin complex, the drug absorption is conditioned by the equilibrium between the free and complexed drug (Fromming, 1987; Uekama and Otagiri, 1987). Therefore, the effect of cyclodextrin complexation on drug absorption is largely dependent upon the magnitude of the stability constant $\left(K_{\mathrm{s}}\right)$ as well as the solubility and dissolution rate of the inclusion complex. This overall process can be described by the scheme shown in Fig. 2 proposed by Uekama and Otagiri (1987). In this scheme, the rate constant for the dissolution of the solid complex and stability constant of the complex in the fluids at the absorption side are represented by $K_{\mathrm{d}}$ and $K_{\mathrm{c}}$, respectively; $K_{\mathrm{a}}$ is the rate constant for the passage of the free form of the drug across the biomembrane. Obviously, the free drug concentration that may be available for absorption is greater when the complex has a smaller stability constant. The complex with a very small stability constant is diluted in the biological fluids after administration, which results in a quick dissociation of the complex with little or no effect on its overall absorption properties. On the contrary, if the complex is very stable or the cyclodextrin concentration is sufficiently high, the equilibrium is shifted greatly toward complexation and the absorption is considered hindered. In the present study, since the $K_{\mathrm{s}}$ values of complexes are relatively low and of the same magnitude (195.7 and $144.8 \mathrm{M}^{-1}$ to $\mathrm{TBM} / \beta-\mathrm{CD}$ and TBM/HP- $\beta-\mathrm{CD}$ complexes, respectively) (Veiga et al., 1996), it seems very unlikely that they have some negative influence in complex dissociation. Then, we can conclude that the solubility, dissolution rate and $K_{\mathrm{s}}$ magnitude of the complexes are not the only limiting factors of TMB absorption.

Simultaneously with the determination of TBM plasma levels, the corresponding hypoglycaemic effects were monitored up to $24 \mathrm{~h}$. The hypoglycaemic response, expressed as glucose variation coefficient (GVC), and the pharmacodynamic parameters are presented in Fig. 3 and Table 3, respectively.

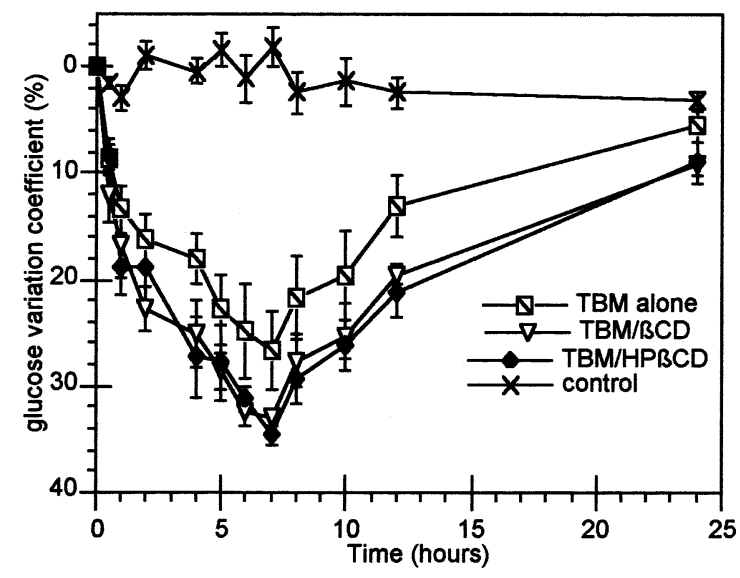

Fig. 3. Glucose variation coefficient-time profiles after administration of TBM, TBM/ $\beta-\mathrm{CD}$ and TBM/HP- $\beta-\mathrm{CD}$ inclusion complexes (equivalent to TBM $20 \mathrm{mg} / \mathrm{kg}$ ) to rabbits $(n=6)$. Each point represents the mean \pm S.D. 
The GVC profiles, like TBM plasma profiles, were practically coincident for $\beta-C D$ and $H P-\beta-$ $\mathrm{CD}$ inclusion complexes. In the absence of CDs, the TBM hypoglycaemic effect was observed with a maximum of $30.55 \%\left(\mathrm{CVG}_{\max }\right)$ at $5.7 \mathrm{~h}\left(T g_{\max }\right)$, after administration of TBM alone. Although the $\mathrm{CVG}_{\max }$ was increased upon $\mathrm{CD}$ complexation $(34.07 \pm 1.01$ and $37.67 \pm 1.48 \%$ to $\beta-C D$ and HP- $\beta-C D$ inclusion complexes, respectively), only the enhancement caused by HP- $\beta-C D$ was statistically significant. The analysis of variance of the areas above the glucose variation coefficient versus time $\left(\mathrm{AAC}_{0-24}\right)$ demonstrated that there was a significant difference between the $\mathrm{AAC}_{0-24}$ of TBM alone and the inclusion complexes. However, the $\mathrm{AAC}_{0-24}$ ratio of the hypoglycaemic effect between the complexes and TBM (about 1.4) was smaller than the corresponding ratio $\left(\mathrm{AUC}_{0-24}\right)$ of TMB plasma levels (about 1.6).

The mean values of $T g_{\max }$ were greater for $\mathrm{TBM} / \beta-\mathrm{CD}$ inclusion complexes than for pure drug and TBM/HP- $\beta-\mathrm{CD}$, but statistical analysis showed that this difference was not significant. Since $T g_{\max }$ values were similar in the three systems, the pharmacodynamic response was not dependent on TBM absorption rate.

The enhanced hypoglycaemic effect displayed by the improvement of TBM bioavailability revealed that there was a correlation between the TBM plasma levels and the hypoglycaemic response. This correlation is clearly evident by comparing Fig. 2 and Fig. 4, in which one seems to be the mirror image of the other. The complexation with cyclodextrins promoted an enhanced and

Table 3

Pharmacodynamic parameters of TBM after oral administration of TBM, TBM/ $\beta-\mathrm{CD}$ and TBM/HP- $\beta-\mathrm{CD}$ inclusion complexes (equivalent to $\mathrm{TBM} 20 \mathrm{mg} / \mathrm{kg}$ ) to rabbits ${ }^{\mathrm{a}}$

\begin{tabular}{lcll}
\hline System & $\mathrm{GVC}_{\max }(\%)$ & $T g_{\max }(\mathrm{h})$ & $\begin{array}{l}\mathrm{AAC}_{0-24} \\
(\% \mathrm{~h})\end{array}$ \\
\hline $\mathrm{TBM}$ & $30.55 \pm 2.94$ & $5.67 \pm 0.80$ & $336.70 \pm 54.16$ \\
$\begin{array}{l}\beta-\mathrm{CD} \\
\quad \text { complex }\end{array}$ & $34.07 \pm 1.01$ & $6.50 \pm 0.81$ & $460.02 \pm 20.10$ \\
$\begin{array}{c}\text { HP- } \beta-\mathrm{CD} \\
\text { complex }\end{array}$ & $37.67 \pm 1.48$ & $5.12 \pm 0.48$ & $471.03 \pm 22.70$ \\
\hline
\end{tabular}

\footnotetext{
${ }^{\text {a }}$ Each value represents the mean \pm S.D. for six rabbits.
}

faster TBM oral absorption, but only increased the hypoglycaemic effect, without any influence on its rate. The absence of proportional relation between TMB plasma concentration and pharmacodynamic effect was also observed by Balant (1981). For this reason, it is common that in TBM and other sulphonylurea studies, the pharmacodynamic effect is evaluated instead of the determination of drug plasma concentrations (Vila-Jato et al., 1987; Kedzierewicz et al., 1993; Torres-Labandeira et al., 1994; Babu and Pankit, 1995).

\section{Conclusion}

Reference TBM demonstrated its poor pharmacokinetic and bioavailability performance following oral administration. Administration of the TBM inclusion complexes was characterised by an oral absorption pattern that was faster and more efficient than that of TBM alone. A correlation between TBM plasma levels and hypoglycaemic effect was achieved. The pharmacodynamic evaluation clearly indicated that TBM hypoglycaemic activity was promoted when complexed with CDs, although without affecting the time for reaching the maximum effect.

The enhanced bioavailability and corresponding potentiation of hypoglycaemic activity by $\mathrm{CD}$ complexation of TBM suggests a dose decrease in oral sulphonylurea therapy with reduction of side effects. With the growing acceptance of CDs as excipients and the presence of CD in formulations rapidly approaching regulatory approval, these pharmacokinetic and pharmacodynamic studies might be beneficial in generating dosage forms not only for TBM but also for a variety of other drugs (Brewster et al., 1997).

\section{References}

Ayres, J., Huang, H., Albert, K., 1984. Generic tolbutamide tablet dissolution, intralot and inter-lot variation. J. Pharm. Sci. 73, 1629-1633.

Babu, R., Pankit, J., 1995. Enhancement of dissolution rate and hypoglycaemic activity of glibenclamide with $\beta$-cyclodextrin. Stp Pharma Sci. 5, 196-201. 
Balant, L., 1981. Clinical pharmacokinetics of sulphonylurea hypoglycaemic activity of glibenclamide drugs. Clin. Pharmacokinet. 6, 215-241.

Brewster, M., 1991. Parental safety and applications of 2-hydroxypropyl- $\beta$-cyclodextrin. In: Duchêne, D. (Ed.), New Trends in Cyclodextrin and Derivatives. Editions de Santé, Paris, pp. 313-351.

Brewster, M., Anderson, W., Menisma, D., Moreno, D., Webb, A., Pablo, L., Estes, K., Derendorf, H., Bodor, N., Sawchuk, R., Cheung, B., Pop, E., 1997. Intravenous and oral pharmacokinetic evaluation of a 2-hydroxypropyl- $\beta$ cyclodextrin-based formulation of carbamazepine in the dog: comparison with commercially available tablets and suspensions. J. Pharm. Sci. 86, 335-339.

Carminetsky, S., 1963. Substitution for brand-name drugs. Can. Med. Assoc. J. 88, 950-955.

Dhanaraju, M., Kumaran, K., Baskaran, T., Moorthy, M., 1998. Enhancement of bioavailability of griseofulvin by its complexation with $\beta$-cyclodextrin. Drug Dev. Ind. Pharm. 24, 583-587.

Fromming, K., 1987. Cyclodextrins in drug formulations. In: Breimer, D., Speiser, P. (Eds.), Topics in Pharmaceutical Sciences. Elsevier, Amsterdam, pp. 169-180.

Kedzierewicz, F., Zinutti, C., Hoffman, M., Maincent, P., 1993. Bioavailability study of tolbutamide $\beta$-cyclodextrin inclusion compounds, solid dispersions and bulk powder. Int. J. Pharm. 94, 69-74.

Miralles, M.J., McGinity, J.W., Martin, A., 1982. Combined water-soluble carriers for coprecipitates of tolbutamide. J. Pharm. Sci. 71, 302-304.

Mura, P., Faucci, M.T., Parrina, P.L., Furlanetto, S., Pinzauti, S., 1999. Influence of the preparation method on the physicochemical properties of ketoprofen-cyclodextrin binary systems. Int. J. Pharm. 179, 117-128.

Özdemir, N., Ordu, S., 1998. Improvement of dissolution properties of furosemide by complexation with $\beta$-cyclodextrin. Drug Dev. Ind. Pharm. 24, 19-25.
Pitha, J., Pitha, J., 1985. Amorphous water-soluble derivatives of cyclodextrins: nontoxic dissolution enhancing excipients. J. Pharm. Sci. 74, 987-990.

Soliman, O.A.E., Kimura, K., Hirayama, F., Uekama, K., El-Sabbagh, H.M., El-Gawad, A., Hashim, F.M., 1997. Amorphous spironolactone-hydroxypropylated cyclodextrin complexes with superior dissolution and oral bioavailability. Int. J. Pharm. 149, 73-83.

Szejtli, J., 1990. Cyclodextrins, properties and applications. Drug Invest. 2 (4), 11-21.

Tillement, J., 1983. The binding of drug to blood plasma macromolecules: recent advances and therapeutic significance. Adv. Drug Res. 13, 75-76.

Torres-Labandeira, J., Blanco-Mendez, J., Vila-Jato, J., 1994. Biopharmaceutical stability of the glibonuride/ $\beta$-cyclodextrin inclusion complex after one year of storage. Stp Pharma Sci. 4, 235-239.

Trinder, P., 1969. Determination of glucose in blood using glucose oxidase with an alternative oxygen acceptor. Ann. Clin. Biochem. 6, 24-28.

Uekama, K., Otagiri, M., 1987. Cyclodextrins in drug carrier systems. CRC Crit. Rev. Ther. Drug Carrier Syst. 3, 1-40.

Varley, A., 1968. The generic inequivalence of drugs. JAMA 206, 1745-1754.

Veiga, F., Teixeira-Dias, J., Kedzierewicz, F., Sousa, A., Maincent, P., 1996. Inclusion complexation of tolbutamide with $\beta$-cyclodextrin and hydroxypropyl- $\beta$-cyclodextrin. Int. J. Pharm. 129, 63-71.

Ventura, C.A., Tirendi, S., Puglisi, G., Bousquet, E., Panza, L., 1997. Improvement of water solubility and dissolution rate of ursodeoxycholic acid and chenodeoxycholic by complexation with natural and modified $\beta$-cyclodextrins. Int. J. Pharm. 149, 1-13.

Vila-Jato, J., Blanco, J., Torres, J., 1987. Biopharmaceutical aspects of the glibornuride- $\beta$-cyclodextrin inclusion compound. Stp Pharma Sci. 3, 28-32.

Vila-Jato, J.L., Blanco, J., Torres, J.J., 1988. Biopharmaceutical aspects of the tolbutamide- $\beta$-cyclodextrin inclusion compound. Il Farmaco 43, 37-45. 